\title{
Solid state MXene based electrostatic fractional capacitors
}

Cite as: Appl. Phys. Lett. 114, 232903 (2019); https://doi.org/10.1063/1.5094236

Submitted: 28 February 2019. Accepted: 29 May 2019. Published Online: 12 June 2019

Shaobo Tu, Qiu Jiang, Xixiang Zhang (D), and Husam N. Alshareef (iD)

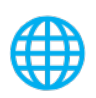

\section{ARTICLES YOU MAY BE INTERESTED IN}

Two-dimensional $\mathrm{MoS}_{2}$ negative capacitor transistors for enhanced (super-Nernstian) signalto-noise performance of next-generation nano biosensors

Applied Physics Letters 114, 233102 (2019); https://doi.org/10.1063/1.5097828

Direct evidence of Mg diffusion through threading mixed dislocations in GaN p-n diodes and its effect on reverse leakage current

Applied Physics Letters 114, 232105 (2019); https://doi.org/10.1063/1.5097767

Memristor effect in $\mathrm{GeO}\left[\mathrm{SiO}_{2}\right]$ and $\mathrm{GeO}[\mathrm{SiO}]$ solid alloys films

Applied Physics Letters 114, 233104 (2019); https://doi.org/10.1063/1.5079690

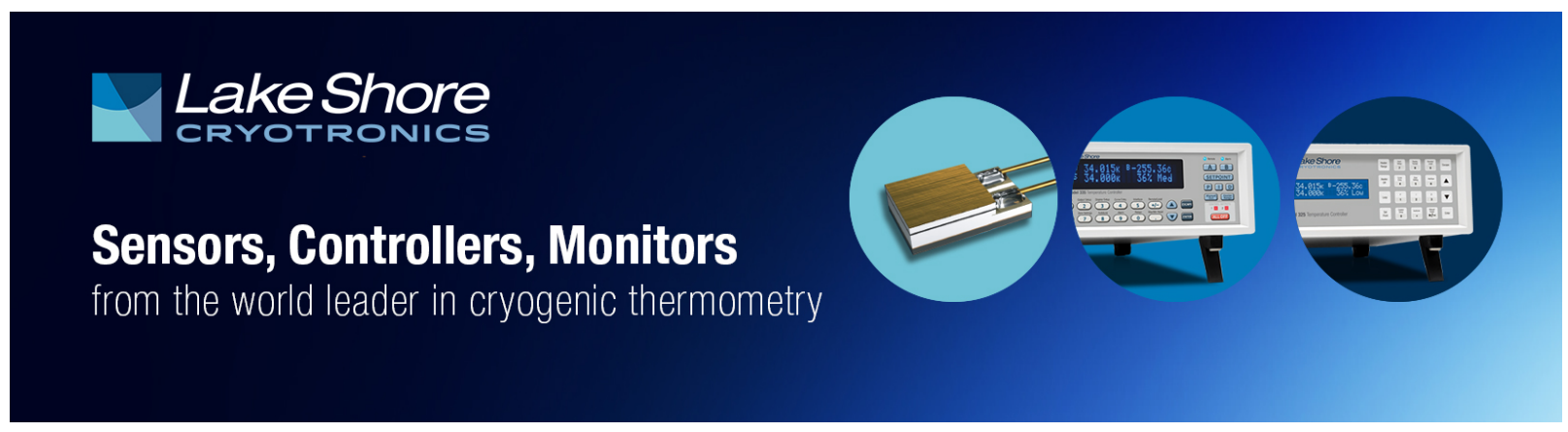




\title{
Solid state MXene based electrostatic fractional capacitors
}

\author{
Cite as: Appl. Phys. Lett. 114, 232903 (2019); doi: 10.1063/1.5094236 \\ Submitted: 28 February 2019 - Accepted: 29 May 2019 . \\ Published Online: 12 June 2019

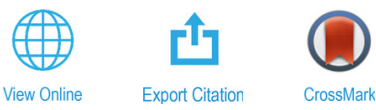

Shaobo Tu, Qiu Jiang, Xixiang Zhang, ${ }^{\text {a) }}$ (D) and Husam N. Alshareef ${ }^{\text {a) }}$ (D)

\section{AFFILIATIONS}

Materials Science and Engineering, King Abdullah University of Science and Technology (KAUST), Thuwal 23955-6900, Saudi Arabia

${ }^{a)}$ Electronic addresses: xixiang.zhang@kaust.edu.sa and husam.alshareef@kaust.edu.sa

\begin{abstract}
Here, we report the fabrication of electrostatic fractional capacitors based on two-dimensional $\mathrm{Ti}_{3} \mathrm{C}_{2}$ MXene/vinylidene fluoride-trifluoroethylene-chlorofluoroethylene terpolymer [P(VDF-TrFE-CFE)] composites. The bandwidth of MXene-based fractional capacitors varies between $200 \mathrm{kHz}$ and $2 \mathrm{MHz}$, while the phase angle varies less than $\pm 2^{\circ}$. Additionally, the constant phase angle of the MXene/P(VDF-TrFE$\mathrm{CFE}$ ) can be precisely tuned from $-67^{\circ}$ to $-34^{\circ}$ by varying the volume ratio of MXene nanosheets in the polymer matrix. The results presented in this work demonstrate the potential of MXene/P(VDF-TrFE-CFE) composites as solid-state electrostatic fractional capacitors.
\end{abstract}

Published under license by AIP Publishing. https://doi.org/10.1063/1.5094236

Inductors, resistors, and capacitors can be classified as passive electrical circuit elements, whose impedance $\mathrm{Z}$ can be expressed in the frequency domain

$$
Z(j \omega)=D(j \omega)^{-\alpha},
$$

where $D$ is the constant coefficient, $\omega$ is the cyclic frequency, $j$ is the unit imaginary number, and $\alpha$ represents the phase angle of $Z$, which is ideally $-1,0$, or 1 . The magnitude and phase angle of this impedance are $|Z|=D / \omega^{\alpha}$ and $\varphi=-\alpha \pi / 2$, respectively. The impedance can be also described in the Laplace domain by substituting $s$ for $j \omega$, and the impedance then becomes

$$
Z(s)=D s^{-\alpha} \text {. }
$$

Three integer values of $\alpha,-1,0$, and 1 , represent the phase angle of $\pi / 2,0$, and $-\pi / 2$ and lead to a pure inductor, pure resistor, or pure capacitor, respectively. ${ }^{1-3}$ If $\alpha$ takes noninteger values, the element is termed as a fractional-order element (FOE). As such, a fractional order element (FOE) exhibits a constant phase behavior and is often referred to in the literature as a constant phase element (CPE). The concept of fractional-order element provides increased flexibility in modeling, characterizing, and understanding the behavior of various electrical circuits and systems leading to several unconventional properties that cannot be achieved using traditional circuit elements. ${ }^{4-14}$ Moreover, fractional order elements offer unique benefits in electronic circuitry by enabling broader impedance matching ${ }^{15,16}$ and allowing flexibility in shaping the frequency response of electronic filters. ${ }^{2,17,18}$ Though many physical processes observed the presence of the fractional order element, realization of such a discrete element is not easy. There are several types of fractional order capacitors $(0<\alpha$ $<1,-90<\varphi<0)$ reported in the literature, including electrochemical capacitors, ${ }^{19}$ fractal structures, ${ }^{20}$ RC ladders, ${ }^{21}$ CMOS-based emulators, ${ }^{22,23}$ polymer dilectrics, ${ }^{2}$ and composites. ${ }^{1,24}$

Two-dimensional materials have attracted intense attention due to their promising potentials in electrical applications. ${ }^{26}$ For the composite fractional order capacitors (FOCs), ${ }^{2}$ in order to represent the response of a RC network, solid-state structures were used to realize FOCs by embedding two-dimensional nanosheets, i.e., reduced graphene oxide $(\mathrm{rGO})^{1}$ sheets or multiwalled carbon nanotubes (MWCNTs) ${ }^{25}$ in the $\mathrm{P}(\mathrm{VDF})$-based polymer matrix. The magnitude of the constant phase angle (CPA) could be tuned by varying the filler volume. Recently, MXene, a $2 \mathrm{D}$ transition metal carbide, was shown to be an effective filler to increase the dielectric constant of the MXene-polymer composites. ${ }^{27}$ However, there have been no attempts to fabricate solid-state fractional order capacitors using MXene fillers.

In this work, we demonstrate that a two-dimensional $\mathrm{Ti}_{3} \mathrm{C}_{2} \mathrm{~T}_{\mathrm{x}}$ MXene/vinylidene fluoride-trifluoroethylene-chlorofluoroethylene terpolymer [P(VDF TrFE CFE)] nanocomposite can be used to fabricate compact and stable electrostatic fractional-order capacitors. The MXenebased fractional order capacitors exhibited a constant phase angle (CPA) that could be tuned from $-67^{\circ}$ to $-34^{\circ}$ by changing the MXene nanosheet loadings over a frequency from $200 \mathrm{kHz}$ to $2 \mathrm{MHz}$.

Exfoliated MXene nanosheets with a $4 \mu \mathrm{m}$ lateral size were prepared using an etching and delamination process. ${ }^{28}$ Figure 1(a) shows the typical transition electron microscopy image of single-layer 
(a)
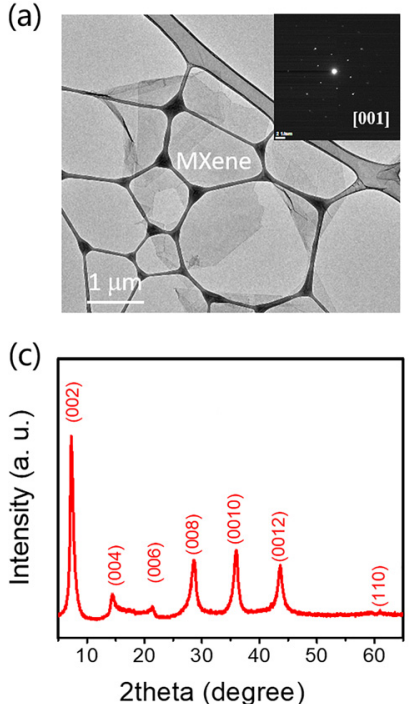

(b)

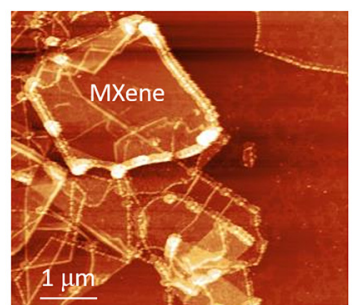

(d)

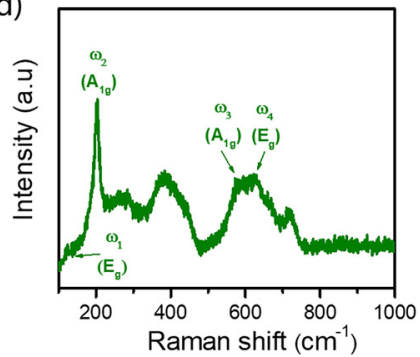

FIG. 1. (a) TEM image of MXene sheets. (b) AFM image of MXene sheets on the mica substrate. (c) and (d) X-ray diffraction and Raman spectroscopy of the $\mathrm{Ti}_{3} \mathrm{C}_{2}$ MXene film.

$\mathrm{Ti}_{3} \mathrm{C}_{2} \mathrm{~T}_{\mathrm{x}}$ sheets. The Fourier transform of the TEM image [inset of Fig. $2(\mathrm{a})$ ] indicates a clear hexagonal symmetry of the $\mathrm{Ti}_{3} \mathrm{C}_{2} \mathrm{~T}_{\mathbf{x}}$ MXene nanosheet. The surface morphology and lateral size of MXene nanosheets are further confirmed by AFM imaging, as shown in Fig. 1(b). Raman spectroscopy and X-ray diffraction (XRD) were used to characterize the MXene sheets. $\mathrm{Ti}_{3} \mathrm{C}_{2} \mathrm{~T}_{\mathrm{x}}$ presents a strong (002) XRD peak at $2 \theta=7.2^{\circ}$ with $\mathrm{FWHM}=0.55^{\circ}$ [see Fig. 1 (c)]. As shown in Fig. 1(d), the Raman spectrum of $\mathrm{Ti}_{3} \mathrm{C}_{2} \mathrm{~T}_{\mathrm{x}} / \mathrm{CF}$ exhibits strong peaks at 205 and $721 \mathrm{~cm}^{-1}$, which can be assigned to the Alg modes of $\mathrm{Ti}_{3} \mathrm{C}_{2} \mathrm{O}_{2}$;

(a)
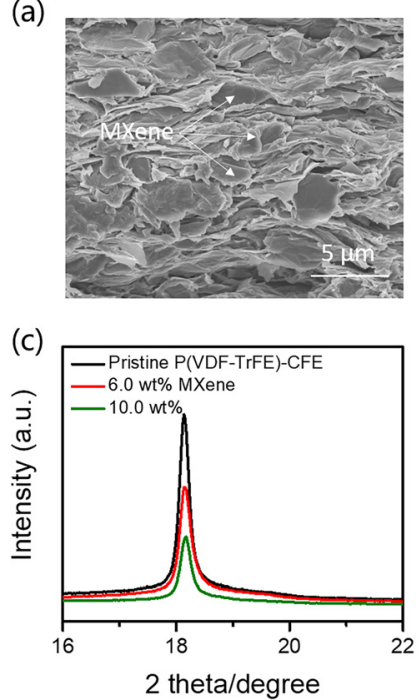

(b)

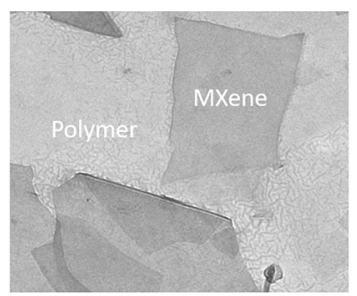

(d)

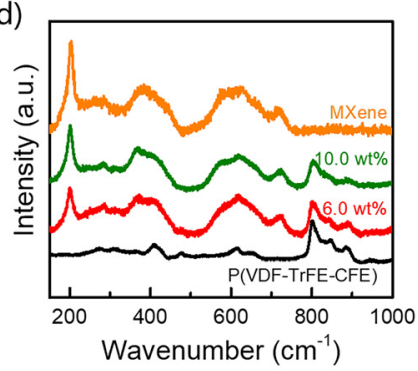

FIG. 2. (a) and (b) SEM and TEM cross-sectional image of the MXene/P(VDFTrFE-CFE) composite with 10.0 wt. \% MXene loading. (c) and (d) Diffraction peak and Raman spectra of the MXene/P(VDF-TrFE-CFE) composite with different MXene loadings.

the additional peaks at 280 , and $620 \mathrm{~cm}^{-1}$ can be assigned to the following vibrational modes: $\mathrm{E}_{\mathrm{g}}$ of $\mathrm{Ti}_{3} \mathrm{C}_{2}(\mathrm{OH})_{2}$, and $\mathrm{E}_{\mathrm{g}}$ of $\mathrm{Ti}_{3} \mathrm{C}_{2} \mathrm{~F}_{2}$, respectively. These peaks reveal the presence of $-\mathrm{OH},-\mathrm{O}$, and $-\mathrm{F}$ functional groups on the $\mathrm{Ti}_{3} \mathrm{C}_{2} \mathrm{~T}_{\mathrm{x}}$ MXene surface. ${ }^{29}$

Figure 2(a) shows cross-sectional SEM image of the MXene/ $\mathrm{P}$ (VDF-TrFE-CFE) composite film, which clearly exhibits a layered morphology (14.0 wt. \%). The TEM image of the MXene/P(VDFTrFE-CFE) composite in Fig. 2(b) indicates that a uniform dispersion of MXene nanosheets in the P(VDF-TrFE-CFE) matrix has been formed. Figure 2(c) shows XRD results for polymer composites containing different MXene loadings, which indicates that the polymer characteristic peak around $18^{\circ}$ decreases as the MXene concentration increases. The MXene concentration in the polymer matrix was investigated by using thermogravimetric analysis (TGA), as shown in Fig. S1. Raman spectra of MXene, P(VDF-TrFE-CFE), and their composites were obtained as shown in Fig. 2(d). It can be seen that the characteristic peaks of the composites consist only of known peaks from pristine MXene and terpolymer, suggesting that there are no reactions or any vibrational changes during the mixing process. As shown in Fig. 2(d), the intensity of the major MXene peak at $205 \mathrm{~cm}^{-1}$ increases with higher MXene loading, while the intensity of the terpolymer peak at $801 \mathrm{~cm}^{-1}$ decreases as the MXene concentration increases.

Figure 3 shows a schematic representation of the fractional order capacitor. As can be seen in Fig. 3(a), the capacitor comprises two electrodes representing the capacitor terminals (bottom and top), a polymer backbone serving as the dielectric, and MXene sheets that reside within the polymer matrix. The presence of conductive MXene sheets, if properly dispersed, can create several capacitors that are connected in series $(\mathrm{Cm} 1, \mathrm{Cm} 2, \ldots)$, as shown in Fig. 3(a), which can be equivalently represented as $\mathrm{C} 1$. Practically, a dielectric loss will exist, which can be modeled as a series resistor (R1). The latter situation, i.e., a capacitor connected in series with a resistor, can be used repetitively to describe the rest of the structure of the capacitor, as shown in Fig. 3(b). Ultimately, the overall capacitor can be modeled as a network very similar to a parallel-combination of series connected Rs and Cs, and the fractional impedance of the network can be tuned by varying the MXene loading. Figure 3(c) shows the impedance $Z(j \omega)$ loci of integer and fractional order $R C$ circuits. For the integer RC circuit, $\alpha=1, \angle Z(j \omega)=-90^{\circ}$, and for fractional order one, $0<\alpha<1$, $-90^{\circ}<\angle Z(j \omega)<0^{\circ}$.

In order to evaluate the performance of fabricated capacitors, the FOCs were characterized using an Agilent 4980A LCR meter in the frequency range between $10 \mathrm{kHz}$ and $2 \mathrm{MHz}$. In total, four fractional capacitors were fabricated, in which the MXene loading varied from 2.0 wt. \% up to 14.0 wt. \%. As can be seen from Fig. 4(a), the phase angle is nearly constant and stable over the frequency range from $200 \mathrm{kHz}$ to $2 \mathrm{MHz}$. Furthermore, we notice that the constant phase angle in the $200 \mathrm{kHz}-2 \mathrm{MHz}$ range increases by introducing the conductive $\mathrm{Ti}_{3} \mathrm{C}_{2} \mathrm{~T}_{\mathrm{x}}$ MXene fillers into the $\mathrm{P}(\mathrm{VDF}-\mathrm{TrFE}-\mathrm{CFE})$ matrix. This phenomenon can be explained by the interplay of MaxwellWagner-Sillars relaxation and dipolar relaxation., ${ }^{2,30}$ The linear relationship between real and imaginary parts of the impedance of the FOCs fabricated using MXene/P(VDF-TrFE-CFE) composites is provided in Fig. 4(b). The slopes of these linear relations are 0.72, 0.69, 0.62 , and 0.37 , and their corresponding CPA values are $-64.8^{\circ}$, $-62.1^{\circ},-55.8^{\circ}$, and $-33.3^{\circ}$, respectively. These values are in agreement with the CPA values obtained by averaging (see Table S1 in the 
(a)

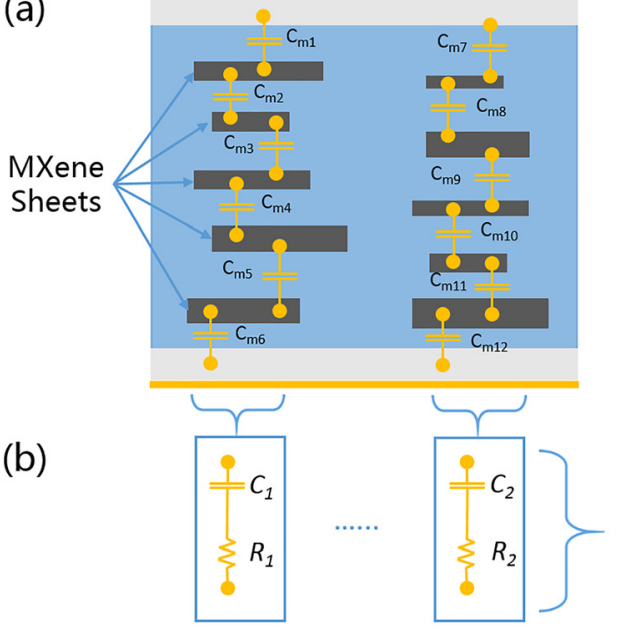

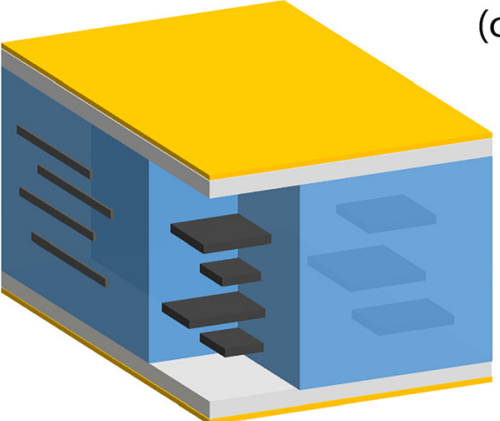

(c)

\section{Circuit Z plane I-V curve}

Integer order RC circuit

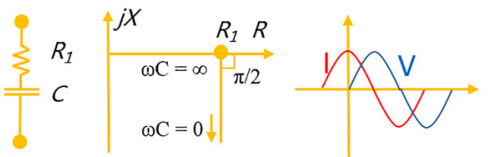

Fractional order RC circuit

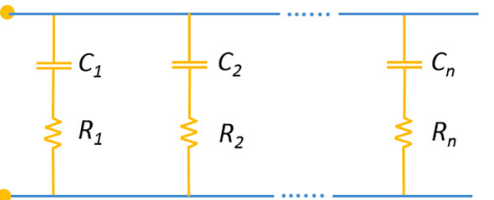

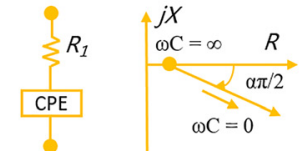

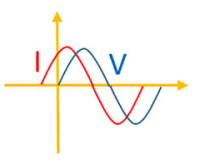

FIG. 3. (a) A conceptual front view and a 3D schematic of the proposed fractional capacitor showing the top and bottom electrodes, the polymer matrix, and the MXene sheets. (b) The approximate equivalent network comprising an equivalent capacitance $(C 1, C 2, \ldots)$ in series with a resistor that models loss $(\mathrm{R} 1, \mathrm{R} 2, \ldots, \mathrm{Rn})$. Horizontal fringing

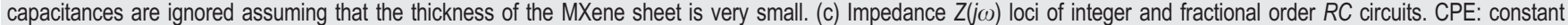
phase element.

supplementary material). As for the fractional capacitor proposed herein, the tunable CPA range is wide enough and the variation is within $\pm 2^{\circ}$. Since the design process allows creating a compact device, integration with other microelectronic circuits/systems and printed circuit boards becomes viable. Furthermore, the proposed capacitors are electrostatic capacitors rather than electrochemical capacitors, which eliminates the hazards associated with chemical spills and electrode corrosion from liquid electrolytes. We also note that percolation allows flexibility in changing the value of the phase angle by changing the loading of the filler material.

In summary, we demonstrate solid-state fractional capacitors fabricated using MXene/P(VDF)-based polymer composites. The fractional capacitors exhibit a tunable but stable phase angle over a broad frequency range. The proposed compact capacitors possess dimensions in the micrometer range enabling integration with a broad range of electronic devices. The percolated MXene-based composites are composed of a large number of microcapacitors, whose phase angle
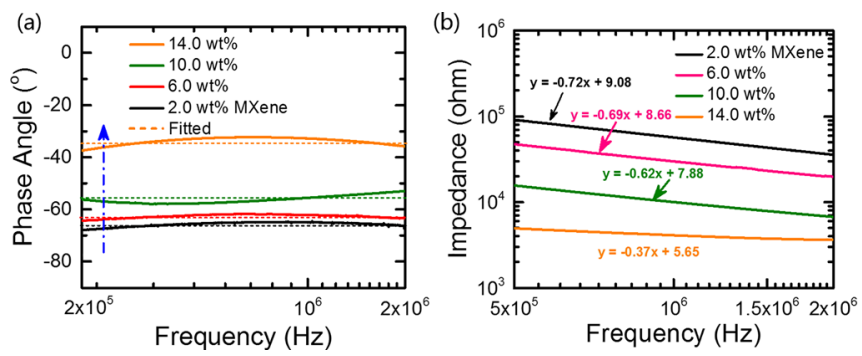

FIG. 4. (a) The measured phase angle of impedance vs frequency from $200 \mathrm{kHz}$ up to $2 \mathrm{MHz}$ for different MXene loadings. (b) The impedance magnitude vs frequency on a log scale. The slope in the line equations represents alpha, and the results confirm the measurement provided in (a). Explicitly, the values of alpha are approximately $0.38,0.60,0.70$, and 0.72 . can be changed by varying the loading of the MXene filler. Our MXene-based approach is an effective method for the realization of surface-mount fractional devices that will ultimately be used in commercial electronics.

See the supplementary material for the thermogravimetric analysis of MXene/P(VDF-TrFE-CFE) composite and the phase angle and corresponding $\alpha$ of MXene/P(VDF-TrFE-CFE) composites with different MXene loadings. The experimental section describes the preparation of MXene and composites and the characterization of devices.

The research reported in this publication was supported by King Abdullah University of Science and Technology (KAUST). The authors would like to thank the Advanced Nanofabrication, Imaging and Characterization Laboratory at KAUST for their excellent assistance.

\section{REFERENCES}

${ }^{1}$ A. M. Elshurafa, M. N. Almadhoun, K. N. Salama, and H. N. Alshareef, Appl. Phys. Lett. 102, 232901 (2013).

${ }^{2}$ A. Agambayev, S. P. Patole, M. Farhat, A. Elwakil, H. Bagci, and K. N. Salama,

ChemElectroChem 4, 2807-2813 (2017).

${ }^{3}$ A. Elwakil, IEEE Circuits Syst. Mag. 10, 40-50 (2010).

${ }^{4}$ A. G. Radwan, A. M. Soliman, and A. S. Elwakil, J. Circuits Syst. Comput. 17, 55-66 (2008).

${ }^{\mathbf{5}}$ A. Adhikary, S. Sen, and K. Biswas, IEEE Trans. Circuits Syst. I 63, 1142-1151 (2016).

${ }^{6}$ A. S. Elwakil, A. Agambayev, A. Allagui, and K. N. Salama, Chaos, Solitons Fractals 96, 160-164 (2017).

${ }^{7}$ L. Said, A. G. Radwan, A. H. Madian, and A. M. Soliman, J. Circuits Syst. Comput. 26, 1750160 (2017).

${ }^{8}$ H. J. In, S. Kumar, Y. Shao-Horn, and G. Barbastathis, Appl. Phys. Lett. 88, 083104 (2006).

${ }^{9}$ A. N. Morozovska, E. A. Eliseev, and S. V. Kalinin, Appl. Phys. Lett. 96, 222906 (2010). 
${ }^{10}$ T. J. Freeborn, B. Maundy, and A. S. Elwakil, IEEE Trans. Emerg. Sel. Topics Power Electron. 3, 367-376 (2013).

${ }^{11} \mathrm{~L}$. Hu, Y. Ren, H. Yang, and Q. Xu, ACS Appl. Mater. Interfaces 6, 14644-14652 (2014).

${ }^{12}$ B. Put, P. M. Vereecken, J. Meersschaut, A. Sepulveda, and A. Stesmans, ACS Appl. Mater. Interfaces 8, 7060-7069 (2016).

${ }^{13}$ A. S. Elwakil and B. Maundy, Electron. Lett. 46, 1367 (2010).

${ }^{14}$ I. Podlubny, I. Petráš, B. M. Vinagre, P. O’Leary, and L'. Dorčák, Nonlinear Dyn. 29, 281-296 (2002)

${ }^{15}$ A. G. Radwan, A. Shamim, and K. N. Salama, IEEE Microwave Wireless Compon. Lett. 21, 120-122 (2011).

${ }^{16}$ A. Shamim, A. G. Radwan, and K. N. Salama, "Fractional Smith chart theory," IEEE Microwaves Wireless Compon. Lett. 21, 117-119 (2011).

${ }^{17}$ A. Soltan, A. G. Radwan, and A. M. Soliman, Microelectron. J. 43, 818-827 (2012).

${ }^{18}$ A. G. Radwan and K. N. Salama, IEEE Trans. Circuits Syst. I 58, 2388-2397 (2011).

${ }^{19}$ M. S. Krishna, S. Das, K. Biswas, and B. Goswami, IEEE Trans. Electron Devices 58, 4067-4073 (2011).

${ }^{20}$ T. C. Haba, G. Ablart, T. Camps, and F. Olivie, Chaos, Solitons Fractals 24, 479-490 (2005).
${ }^{21}$ S. Roy, IEEE Trans. Circuit Theory 14, 264-274 (1967).

${ }^{22}$ G. Tsirimokou, C. Psychalinos, A. Elwakil, and K. Salama, Electron. Lett. 52, 1298-1300 (2016).

${ }^{23}$ G. Tsirimokou, C. Psychalinos, and A. S. Elwakil, Analog Integr. Circuits Signal Process. 85, 413-423 (2015).

${ }^{24}$ D. A. John, S. Banerjee, G. W. Bohannan, and K. Biswas, Appl. Phys. Lett. 110, 163504 (2017).

${ }^{25}$ A. Agambayev, K. H. Rajab, A. H. Hassan, M. Farhat, H. Bagci, and K. N. Salama, J. Phys. D: Appl. Phys. 51, 065602 (2018).

${ }^{26}$ T. Kobayashi, N. Hori, T. Nakajima, and T. Kawae, Appl. Phys. Lett. 108, 132903 (2016)

${ }^{27}$ S. B. Tu, Q. Jiang, X. X. Zhang, and H. N. Alshareef, ACS Nano 12, 3369-3377 (2018).

${ }^{28}$ M. Naguib, O. Mashtalir, J. Carle, V. Presser, J. Lu, L. Hultman, Y. Gogotsi, and M. W. Barsoum, ACS Nano 6, 1322-1331 (2012).

${ }^{29} \mathrm{~T}$. Hu, J. Wang, H. Zhang, Z. Li, M. Hu, and X. Wang, Phys. Chem. Chem. Phys. 17, 9997-10003 (2015).

${ }^{30}$ G. Zhang, D. Brannum, D. Dong, L. Tang, E. Allahyarov, S. Tang, K. Kodweis, J. K. Lee, and L. Zhu, Chem. Mater. 28, 4646-4660 (2016). 